\title{
Gérer la douleur et la peur : Jouez vos «cartes» pour améliorer l'expérience de vaccination
}

\author{
Anna Taddio ${ }^{1 *}$, Anthony llersich², C Meghan McMurtry ${ }^{3}$, Lucie M Buccii ${ }^{4}$, Noni E MacDonald ${ }^{5}$
}

\section{Résumé}

La plupart des vaccins sont administrés avec une aiguille, ce qui peut provoquer des douleurs et des réactions liées à la douleur, tels que la peur et l'évanouissement. À l'heure actuelle, les interventions visant à prévenir la douleur et les réactions connexes ne sont pas systématiquement intégrées dans le processus d'administration de vaccins, bien qu'elles contribuent aux expériences négatives de la vaccination et à la non-adhésion occasionelle. Cet article examine une nouvelle démarche pour l'administration de vaccins, appelée le système $\mathrm{CARD}^{\mathrm{MC}}$. CARD est l'acronyme de Confort, Aide, Relaxation et Distraction, où chaque lettre est associée à une catégorie d'interventions fondées sur des preuves pour réduire la douleur, la peur et les réactions connexes. Le système CARD peut être intégré dans les activités habituelles de planification et d'administration des vaccinations dans de nombreux contextes afin d'améliorer l'expérience de vaccination et de réduire la douleur et la peur qui font obstacle à la vaccination. Les vaccinateurs de tous les milieux et les responsables des organisations sont invités à revoir leurs services de vaccination par rapport au système CARD afin de cerner les possibilités d'améliorer la qualité des soins fournis.

Citation proposée : Taddio A, Ilersich A, McMurtry CM, Bucci LM, MacDonald NE. Gérer la douleur et la peur Jouez vos «cartes» pour améliorer l'expérience de vaccination. Relevé des maladies transmissibles au Canada 2021;47(1):99-103. https://doi.org/10.14745/ccdr.v47i01a12f

Mots-clés : vaccination, douleur, peur, gestion de la douleur, hésitation à se faire vacciner, piqûres d'aiguille

\section{Introduction}

Les injections de vaccins sont souvent associées à la douleur et à leurs effets indésirables connexes, tels que la peur, l'évanouissement, la nausée et d'autres réactions liées au stress $(1,2)$. Jusqu'à tout récemment, peu d'attention a été accordée à la réduction de la douleur et des réactions connexes au moment de la vaccination. Toutefois, les preuves accumulées montrent que les expériences de vaccination négatives peuvent donner lieu à la peur des aiguilles, à l'hésitation à se faire vacciner et à des comportements d'évitement de soins de santé, y compris le refus de se faire vacciner $(3,4)$. Cela est particulièrement important pendant l'enfance, lorsque les inquiétudes concernant la douleur et la peur des aiguilles sont élevées et que l'attitude envers les professionnels de la santé est en train de se former $(2,5)$.

La vaccination est la situation la plus courante, et de loin, où les gens ont affaire à des aiguilles. L'Organisation mondiale de la Santé a fait de l'élimination des obstacles à la vaccination une priorité pour la santé mondiale (6). Il est encore plus pertinent de s'attaquer aux obstacles à la vaccination maintenant, pendant la pandémie, afin de favoriser l'acceptation du ou des vaccins contre la COVID-19 lorsqu'ils seront disponibles.
Cette oeuvre est mise à la disposition selon les termes de la licence internationale Creative Commons Attribution 4.0

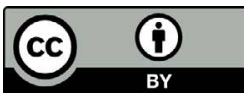

Affiliations

${ }^{1}$ Faculté de pharmacie Leslie Dan, Université de Toronto, Toronto, ON

2 Faculté de mathématiques, Université de Waterloo, Waterloo, ON

${ }^{3}$ Département de psychologie, Université de Guelph, Guelph, ON

${ }^{4}$ Immunisation Canada, Association canadienne de santé publique, Ottawa, ON

${ }^{5}$ Faculté de médecine, Université de Dalhousie, Halifax, NS

${ }^{\star}$ Correspondance :

anna.taddio@utoronto.ca
Les conséquences négatives de la douleur non gérée au moment de se faire piquer avec une aiguille sont nombreuses. Les personnes sont souvent soumises à des procédures plus longues et à un recours accru à l'immobilisation, en plus de faire l'objet d'événements indésirables potentiellement graves tels que des évanouissements, des nausées et d'autres réactions liées au stress $(2,7,8)$. Le fait de devoir faire face à des interactions longues et complexes avec les patients entraîne un stress supplémentaire pour les professionnels de la santé qui administrent les vaccins. Les vaccinateurs font souvent état de difficultés dans les processus actuels de vaccination, qui peuvent augmenter le risque de résultats indésirables. Ces défis comprennent les espaces physiques sous-optimaux, le manque de préparation et de communication au niveau des intervenants clés, les rôles peu clairs, les demandes concurrentes et les symptômes excessifs chez certains patients (peur, douleur, vertige), en particulier chez les enfants (9-11). Récemment, la crainte de contracter une infection par la COVID-19 pendant la vaccination n'a fait qu'exacerber ces préoccupations.

Il existe de nombreuses interventions réalisables et fondées sur des preuves permettant d'améliorer l'expérience de vaccination (1). La gestion de la douleur doit être reconnue 
comme faisant partie des bonnes pratiques de vaccination et ces connaissances doivent être systématiquement intégrées dans la pratique (12). Sur la base de son guide de pratique clinique (1), I'équipe nationale de HELPinKids\&Adults a récemment mis au point un cadre de vaccination, appelé le système CARD ${ }^{M C}$, qui montre aux vaccinateurs et aux responsables de programmes chargés de l'administration des vaccins comment intégrer ces interventions dans les processus de planification et d'administration des vaccinations. En outre, le système CARD apprend aux patients à faire face à leur propre expérience de vaccination (11). Cet article explique le cadre et la manière de le mettre en pratique dans différents contextes.

\section{Qu'est-ce que le système CARD?}

CARD signifie Confort, Aide, Relaxation et Distraction. Chacune des quatre lettres de l'acronyme est associée à une catégorie d'interventions, et ces quatre catégories englobent des activités qui visent à réduire la douleur, la peur, l'évanouissement et les réactions connexes avant, pendant et après la vaccination. Le système CARD peut être mis à profit avec des enfants et des adultes, et au sein d'activités participatives faisant appel aux quatre catégories d'interventions.

\section{Comment le système CARD a-t-il été créé?}

Bien que le système CARD ait été conçu à l'origine pour améliorer l'expérience de vaccination en milieu scolaire, il constitue un outil précieux pour l'administration de vaccins dans divers contextes, y compris dans les bureaux des prestataires de soins de santé. En fait, l'un de ses principaux atouts réside dans son adaptabilité à une foule de contextes différents. Plus important encore, le système CARD a été conçu avec la participation de différents intervenants ayant un rôle à jouer dans les vaccinations en milieu scolaire : les vaccinateurs, les élèves, les parents/tuteurs et le personnel scolaire. Une démarche par étape a été utilisée pour cerner les besoins et les préférences, élaborer des outils et des ressources, et évaluer l'effet de la mise en œuvre (11).

\section{Le système CARD fonctionne}

Lors d'un essai comparatif en grappes mené à Niagara, en Ontario, les élèves des écoles où le système CARD a été mis en œuvre (comparativement aux témoins) ont déclaré éprouver moins de peur (rapport de cotes $[R C]=0,47$, intervalle de confiance $[I C]$ de $95 \%$ ) : de 0,27 à 0,82 ) et d'étourdissements $(\mathrm{RC}=0,26, \mathrm{IC}$ de $95 \%$ : de 0,07 à 0,91 ) pendant la vaccination (13). Les élèves formés au système CARD ont obtenu de meilleurs résultats quant aux connaissances et ont eu une attitude plus positive à l'égard de la vaccination. Les élèves souhaitaient notamment que d'autres élèves se renseignent sur le système CARD, car ils l'avaient trouvé très utile (13). Les vaccinateurs, les parents/tuteurs et le personnel scolaire ont également fait état d'attitudes plus positives à l'égard de l'expérience de vaccination lorsque le système CARD était mis en pratique (14).

\section{Adapter le système CARD à votre environnement}

Le système CARD peut être adapté en fonction de divers environnements, notamment des bureaux privés, des hôpitaux, des écoles et des pharmacies. Les éléments clés du système CARD comprennent l'éducation des vaccinateurs et des patients, la mise en place d'un site de vaccination pour favoriser les interactions entre les vaccinateurs et les patients et garantir que ces dernières tiennent compte des préférences des patients (i.e. les choix qu'offre le système CARD). Les patients peuvent s'informer sur le système CARD grâce à des ressources en ligne, par exemple des vidéos et des brochures (11). Bien que la majorité de ces ressources sont destinées aux adolescents, des ressources sont en cours d'élaboration pour des contextes de vaccination des adultes (https://immunize.ca/fr/card-pourles-adultes). La figure 1 présente des exemples d'interventions que les patients peuvent mettre en pratique pour faire de la vaccination une expérience plus positive.

Les vaccinateurs reçoivent également une formation simple sur l'importance des éléments du système CARD et sur la manière de respecter les choix des patients. Le vaccinateur et le patient forment une équipe de sorte à rendre l'expérience aussi positive que possible. Depuis les étapes de planification jusqu'à l'injection proprement dite, et dans différents contextes de vaccination, le vaccinateur peut comparer les procédures de vaccination courantes avec la démarche que propose le système CARD, en cherchant des possibilités d'intégrer des moyens d'optimiser l'expérience de vaccination.

Le tableau 1 résume certaines des activités normalement associées à la planification et à l'administration des vaccinations et la manière d'intégrer le système CARD dans ces activités. Tous les intervenants participant au processus de vaccination, y compris les vaccinateurs et les patients, peuvent «jouer leurs cartes " pour favoriser une expérience de vaccination plus positive. Par exemple, les vaccinateurs peuvent s'assurer que les patients sont assis confortablement (Confort) et inviter ces derniers à poser des questions avant, pendant et après la vaccination (Aide). Cela permet aux patients de se sentir à l'aise, de sentir qu'ils sont informés et qu'ils participent au processus, ce qui les aide à se sentir calmes. Le système renforce également la confiance dans les prestataires de soins de santé, puisque ces derniers démontrent qu'ils sont attentifs aux besoins des patients. Les vaccinateurs peuvent également poser des questions aux patients (e.g. "Avez-vous peur?», "Préférez-vous ne pas regarder?») pour les aider à évaluer l'état du patient et à faire des patients des participants actifs. Les patients peuvent 
Figure 1 : Exemples d'interventions tirés du système CARD

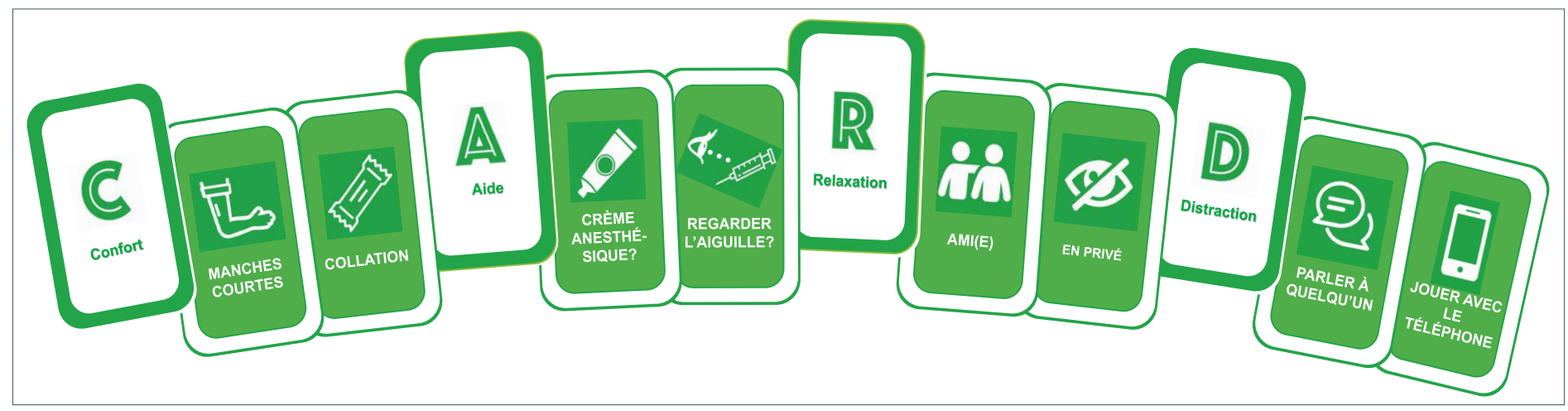

Source : Reproduit avec l'autorisation d'Anna Taddio, professeure, Faculté de pharmacie Leslie Dan, Université de Toronto, Canada

Tableau 1 : Cadre d'administration des vaccinations du système CARD

\begin{tabular}{c|c}
$\begin{array}{c}\text { Phase du } \\
\text { processus de } \\
\text { vaccination }\end{array}$ & Activité du vaccinateur \\
Préparation/planification &
\end{tabular}

Tableau 1 : Cadre d'administration des vaccinations du système CARD (suite)

Phase du
processus de
vaccination $\quad$ Activité du vaccinateur

- Favoriser un environnement calme et être positif

- Vérifier les antécédents médicaux des patients, y compris les épisodes d'évanouissement et le niveau de crainte ou d'inquiétude concernant la vaccination

- Répondre aux questions des patients

- Communiquer en utilisant un langage neutre. Ne pas utiliser pas de mots qui suscitent la peur (e.g. I'aiguille "pique») et ne pas rassurer constamment (e.g. «ne vous inquiétez pas, tout va bien, il ne vous arrivera rien »)

- Fournir des renseignements objectifs. Ne pas faire valoir que la vaccination ne fait pas mal; décrire plutôt les sensations (e.g. "pression» et "pincement») et la durée (e.g. "environ 1 seconde»), et inviter les patients à faire part de leurs sentiments

- Demander aux patients quelles sont leurs préférences. Ne pas imposer de stratégies d'adaptation telles que parler pour distraire, prendre de grandes respirations, détourner le regard pendant l'injection (ces stratégies vont à l'encontre des stratégies d'adaptation préférées

Administration de nombreuses personnes et entraînent une augmentation des niveaux de peur ou de stress)

des vaccins

- Demander aux patients quelles «cartes» du système CARD ils veulent jouer, et respecter leurs choix (e.g. produit anesthésique sur la peau, personne de soutien, espace privé, injection de deux vaccins dans le même bras)

- Fournir des articles favorisant la distraction aux patients qui n'en ont pas et qui en font la demande (conformément aux directives de contrôle et de prévention des infections)

- Demander aux patients quelle est leur préférence en ce qui concerne le bras qui recevra le vaccin. S'il n'y a pas de préférence, injecter le vaccin dans le bras non dominant

- Demander aux patients quelle est leur préférence en ce qui concerne l'injection de deux vaccins dans le même bras

- Encourager les patients à détendre leur bras afin qu'il soit souple et mou

- Songez à ne pas nettoyer la peau avec de l'alcool avant l'injection, vu que cette étape n'est pas nécessaire et qu'elle prendra du temps additionnel et pourrait contribuer à du stress anticipatoire $^{b}$ 


\section{Tableau 1 : Cadre d'administration des vaccinations du système CARD (suite)}

\begin{tabular}{|l|l}
$\begin{array}{c}\text { Phase du } \\
\text { processus de } \\
\text { vaccination }\end{array}$ & \multicolumn{1}{c}{ Activité du vaccinateur } \\
\hline Le jour de la vaccination (suite) \\
\hline & - Vacciner les patients lorsqu'ils sont assis en \\
& $\begin{array}{l}\text { position verticale (sur les genoux d'un parent ou } \\
\text { d'un tuteur si le patient est un jeune enfant) }\end{array}$ \\
- Injecter les vaccins rapidement, sans délai \\
d'attente
\end{tabular}

Ressources/outils disponibles en ligne (11)

boir Organisation mondiale de la Santé. Boîte à outils des meilleures pratiques de l'OMS en matière d'injections et de procédures connexes. Genève (Suisse) : Services de production de documents de l'OMS; 2010 (15)

'Voir l'annexe 5, page 2 de Taddio et al. (1)

Source : Reproduit avec l'autorisation d'Anna Taddio, professeure, Faculté de pharmacie Leslie Dan, Université de Toronto, Canada

apporter un article de leur choix (Confort) ou un appareil électronique (Distraction) dont ils peuvent se servir pendant la vaccination pour les aider à faire face à la situation. Bien que chaque intervenant (vaccinateurs et patients) ait ses propres "cartes» à jouer, le potentiel du cadre qu'offre le système CARD est pleinement réalisé lorsque tous jouent leurs «cartes» préférées dans le but commun d'améliorer l'expérience de vaccination.

\section{Système CARD est travail d'équipe}

Dans de nombreux cas, la vaccination est complexe et les vaccinateurs ne sont pas en mesure d'apporter par eux-mêmes les changements nécessaires favorisant l'adoption du système CARD. II est essentiel de faire participer les dirigeants des organisations car ils sont en mesure d'en faciliter l'adoption en apportant des changements aux politiques et procédures pertinentes. Ces changements pourraient porter sur les rôles du personnel et la formation, et sur la communication et l'évaluation continues (e.g. réunions du personnel, résumés, audit et retour d'information) et le soutien continu (e.g. ressources éducatives) (11). II s'agit notamment de promouvoir la sensibilisation et la compréhension des modèles généraux de prestation de soins de santé et des normes professionnelles qui mettent en valeur les soins axés sur la personne et les pratiques fondées sur des données probantes, et de pratiquer activement l'amélioration continue de la qualité et la pratique réflexive.

Les vaccinateurs et leurs responsables organisationnels peuvent également cerner les possibilités d'intégrer des activités propres au système CARD dans leurs activités courantes. Par exemple, les vaccinateurs informent généralement les enseignants et les parents/tuteurs des vaccinations à venir en milieu scolaire. Ces intervenants peuvent être formés au système CARD de sorte à former à leur tour les enfants au système CARD. La participation des intervenants, notamment des enseignants, des parents et tuteurs, confère de multiples avantages, dont l'amélioration de la fiabilité de la mise en œuvre, la création d'une «norme sociale» qui reconnaît et prend en considération la participation des personnes à l'égard de leurs soins de santé et leurs préférences en matière de renseignements et d'adaptation, ainsi que la réduction des besoins en ressources supplémentaires. En retour, les parents/tuteurs et les enseignants sont rassurés de savoir que les enfants sont pris en charge, ce qui crée un environnement plus accueillant pour tous.

Notre expérience de l'utilisation du système CARD dans un programme de vaccination en milieu scolaire à Niagara, en Ontario, a montré qu'après une formation et un soutien lors de la mise en œuvre initiale, le système pouvait être intégré aux activités habituelles sans coûts supplémentaires (12). Les vaccinateurs auront besoin d'un peu plus de temps pour se préparer aux vaccinations en raison des étapes de planification, telles que la formation à l'intention de tous les intervenants. Cependant, le temps nécessaire diminuera à mesure que chacun se familiarisera avec le système CARD.

\section{Conclusion}

Il est prouvé que la gestion de la douleur et des réactions liées au stress améliore l'expérience de vaccination, tant pour les patients que pour les vaccinateurs. Les avantages à long terme du cadre qu'offre le système CARD sont nombreux et peuvent contribuer à améliorer les résultats en matière de santé grâce à une meilleure acceptation des interventions de soins de santé, y compris la vaccination. Le système CARD permet aux vaccinateurs de «jouer leurs meilleures cartes» en ce qui concerne la mise en place et l'exploitation de cliniques ou la gestion de rendez-vous de vaccination individuels. Le système CARD est un outil précieux pour optimiser l'expérience de vaccination et s'attaquer à l'un des aspects reconnus et parfois négligés de la vaccination, la peur de l'aiguille.

\section{Déclaration des auteurs}

A. T. - Conceptualisation, rédaction-ébauche originale, révision et édition

A. I. - Conceptualisation, rédaction-révision et édition 
C. M. M. - Conceptualisation, rédaction-révision et édition

L. M. B. - Rédaction-révision et édition

N. E. M. - Rédaction-révision et édition

Tous les auteurs assument la pleine responsabilité du contenu de cet article.

\section{Intérêts concurrents}

A. T. signale une marque de commerce dans la section $9 n^{\circ}$ 924835 pour CARD L. M. B. signale qu'Immunisation Canada a reçu des subventions de Pfizer Canada, Merck Canada, GSK Canada, Seqirus Canada et Sanofi Pasteur en dehors des travaux présentés. II n'y a pas d'autres divulgations.

\section{Remerciements}

Les auteurs remercient $M$. Ho pour la création de la figure 1.

\section{Financement}

Ce travail est financé par les Instituts de recherche en santé du Canada (FRN 159905).

Le contenu de l'article et les points de vue qui y sont exprimés n'engagent que les auteurs et ne correspondent pas nécessairement à ceux du gouvernement du Canada.

\section{Références}

1. Taddio A, McMurtry CM, Shah V, Riddell RP, Chambers CT, Noel M, MacDonald NE, Rogers J, Bucci LM, Mousmanis P, Lang E, Halperin SA, Bowles S, Halpert C, Ipp M, Asmundson GJ, Rieder MJ, Robson K, Uleryk E, Antony MM, Dubey V, Hanrahan A, Lockett D, Scott J, Bleeker EV; HELPinKids\&Adults. Reducing pain during vaccine injections: clinical practice guideline. CMAJ 2015;187(13):975-82. DOI PubMed

2. McMurtry CM, Pillai Riddell $R$, Taddio A, Racine $N$, Asmundson GJ, Noel M, Chambers CT, Shah V; HELPinKids\&Adults Team. HELPinKids\&Adults Team. Far from "just a poke": common painful needle procedures and the development of needle fear. Clin J Pain 2015;31(10 Suppl):S3-11. DOI PubMed

3. Taddio A, Ipp M, Thivakaran S, Jamal A, Parikh C, Smart S, Sovran J, Stephens D, Katz J. Survey of the prevalence of immunization non-compliance due to needle fears in children and adults. Vaccine 2012;30(32):4807-12. DOI PubMed

4. MacDonald NE; SAGE Working Group on Vaccine Hesitancy. Vaccine hesitancy: Definition, scope and determinants. Vaccine 2015;33(34):4161-4. DOI PubMed

5. Taddio A, llersich AF, llersich AN, Wells J. From the mouth of babes: getting vaccinated doesn't have to hurt. Can J Infect Dis Med Microbiol 2014;25(4):196-200. DOI PubMed
6. Organisation mondiale de la Santé. Dix ennemis que l'OMS devra affronter cette année. Genève : OMS; 2019 (accédé 2020-12-15). https://www.who.int/fr/news-room/spotlight/ ten-threats-to-global-health-in-2019

7. McMurtry CM. Gestion des réponses liées au stress dans le cadre de la vaccination: Un facteur déterminant pour maintenir la confiance envers les vaccins. Relevé des maladies transmissibles au Canada 2020;46(6):237-45. DOI

8. Gold MS, MacDonald NE, McMurtry CM, Balakrishnan MR, Heininger U, Menning L, Benes O, Pless R, Zuber PL. Immunization stress-related response - Redefining immunization anxiety-related reaction as an adverse event following immunization. Vaccine 2020;38(14):3015-20. DOl PubMed

9. Kikuta A, Gardezi F, Dubey V, Taddio A. Practices and perceptions regarding pain and pain management during routine childhood immunizations: findings from a focus-group study with nurses working at Toronto Public Health, Ontario. Can J Infect Dis Med Microbiol 2011;22(2):43-8. DOI PubMed

10. Gerges S, Peter E, Bowles SK, Diamond S, Bucci LM, Resnick A, Taddio A. Pharmacists as vaccinators: an analysis of their experiences and perceptions of their new role. Hum Vaccin Immunother 2018;14(2):471-7. DOI PubMed

11. Taddio A, McMurtry CM, Bucci LM, MacDonald N, Ilersich AN, Ilersich AL, Alfieri-Maiolo A, deVlaming-Kot C, Alderman L; Pain Pain Go Away Team. Overview of a Knowledge Translation (KT) Project to improve the vaccination experience at school: the CARDTM System. Paediatr Child Health 2019;24 Suppl 1:S3-18. DOI PubMed

12. World Health Organization. Reducing pain at the time of vaccination: WHO position paper - September 2015. Wkly Epidemiol Rec 2015;90(39):505-10. DOI PubMed

13. Freedman $T$, Taddio A, McMurtry CM, Wong $H$, MacDonald N, McDowall T, deVlaming-Kot C, Alderman L; Pain Pain Go Away Team. Involving stakeholders in informing the development of a Knowledge Translation (KT) intervention to improve the vaccination experience at school. Paediatr Child Health 2019 Apr;24 Suppl 1:S19-28. DOI PubMed

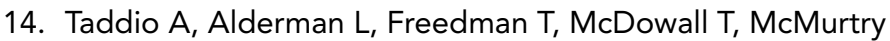
CM, MacDonald N, deVlaming-Kot C, Alfieri-Maiolo A; Pain Pain Go Away Team. The CARD TM System for improving the vaccination experience at school: results of a small-scale implementation project on program delivery. Paediatr Child Health 2019 Apr;24 Suppl 1:S54-67. DOI PubMed

15. World Health Organization. WHO best practices for injections and related procedures toolkit. March 2010. Geneva: WHO; 2010. https://apps.who.int/iris/bitstream/han dle/10665/44298/9789241599252_eng.pdf;jsessionid=BCD6 FA7D110B62AB5289824942EC6294? sequence =1 